\title{
Innovación educativa y programas de inmersión en la enseñanza de lenguas a escolares: propuesta curricular
}

\section{Programas educativos de inovação e imersão no ensino de línguas para alunos: proposta curricular}

Educational innovation and immersion programs in the teaching of languages to schoolchildren: curricular proposal

\author{
María del Carmen Quiles Cabrera ${ }^{1}$
}

DOI: http://dx.doi.org/10.20435/serie-estudos.v23i49.1140

\begin{abstract}
Resumen: El trabajo pretende abordar el concepto de innovación educativa en el ámbito de la enseñanza de lenguas extranjeras. Si bien al hablar de este concepto en la escuela solemos pensar en el uso de herramientas TIC y en la incorporación de ámbito digital, en didáctica de la lengua existen otros elementos que vienen marcados por la innovación y la transferencia del conocimiento, tan necesaria en el momento actual. Retomamos la idea de inmersión lingüística pero entendiéndola desde una perspectiva actual acorde con las exigencias del siglo XXI, para lo que el intercambio y la integración en la comunidad de hablantes en la lengua meta - esto es, en un contexto homóglota se concibe como un pilar básico. En este sentido, tomaremos como referente el proyecto P.A.L.S., promovido por la institución educativa Country-Cousins School (UK) y galardonado con un premio ElTons. En dicho programa educativo, convergen elementos como el aprendizaje servicio, el concepto de comunidad de aprendizaje y la tutoría entre iguales: peer tutoring.
\end{abstract}

Palabras clave: innovación educativa; enseñanza de lenguas extranjeras; aprendizaje-servicio.

Resumo: O artigo pretende abordar o conceito de inovação educacional no campo do ensino de línguas estrangeiras. Ao falar sobre esse conceito na escola, geralmente pensamos sobre o uso de ferramentas de TIC e a incorporação do escopo digital, na didática da linguagem existem outros elementos que são marcados pela inovação e transferência de conhecimento, tão necessário na hora atual. Retornamos à ideia de imersão linguística, mas entendemos isso de uma perspectiva atual de acordo com os requisitos do século XXI, para o qual a troca e integração na comunidade de falantes na língua alvo, ou seja, em um contexto homogêneo é concebida como um pilar básico. A este respeito, tomaremos como referência o projeto P.A.L.S., promovido pela instituição

\footnotetext{
${ }^{1}$ Universidad de Almería, Almería, Espanha.
} 
educacional Country-Cousins School (Reino Unido) e premiado com um prêmio ElTons. Neste programa educacional, elementos como a aprendizagem de serviços, o conceito de comunidade de aprendizado e aulas de pares convergem: peer tutoring.

Palavras-chave: inovação educacional; ensino de línguas estrangeiras; serviço de aprendizagem.

\begin{abstract}
The paper aims to address the concept of educational innovation in the field of foreign language teaching. While talking about this concept in school we usually think about the use of ICT tools and the incorporation of digital scope, in language didactics there are other elements that are marked by innovation and the transfer of knowledge, so necessary in the current time. We return to the idea of linguistic immersion but understand it from a current perspective in line with the requirements of the 21st century, for which the exchange and integration in the target language community, that is, in a homogeneous context is conceived as a basic pillar. In this regard, we will take as reference the project P.A.L.S., promoted by the educational institution Country-Cousins School (UK) and awarded with an ElTons award. In this educational program, elements such as service learning, the concept of learning community and peer tutoring converge.
\end{abstract}

Keywords: educational innovation; teaching of foreign languages; learning-service.

\title{
1 ENSEÑANZA DE LENGUAS, INMERSIÓN E INNOVACIÓN
}

La enseñanza de la lengua en el momento presente ha de mirar constantemente a su alrededor. Ha de afrontar el reto de estar al corriente de las transformaciones sociales y asumir las necesidades de cambio. El concepto de innovación educativa es un marbete que parece haberse puesto de moda. Y no sólo eso, sino que se viene asociando de manera inconsciente al ámbito tecnológico. Tendríamos que preguntarnos si acaso la innovación solo radica en la incorporación de recursos técnicos a la práctica docente. Si la respuesta es afirmativa, tendríamos que aceptar que el uso de un libro de texto a través de la pizarra digital, empleado de la misma manera que venimos haciéndolo en su formato en papel, ya es innovación educativa. Ahora bien, si planteamos una reflexión que vaya más allá, estaremos observando que lo que cambia la perspectiva educativa no es el uso de uno u otro soporte, sino el modo en que planificamos la tarea docente y el enfoque metodológico y epistemológico del que partamos a la hora de trasladar determinado contenido al aula. Si tomamos esta premisa como cierta, defenderemos que la innovación, sobre todo en ciencias como las humanas, puede radicar en proyectos que, de entrada, nada tienen que ver con la tecnología ni con la incorporación de los recursos electrónicos. Se trata de proyectos marcados por la búsqueda de alternativas en el día a día cotidiano, en la realidad más palpable del entorno del aula. 
Un buen ejemplo de ello es el proyecto P.A.L.S. (Promoting Acceptance Language Students) - Proyecto para la integración de estudiantes de lenguas extranjeras-, promovido por el centro Country-Cousins School (U.K.), galardonado con uno de los prestigiosos premios ElTons del British Council, sobre el que hablaremos más adelante. Merece la pena detenernos en este ejemplo de innovación para obtener un modelo de aplicación no sólo en el contexto del inglés como lengua extranjera, sino también para la enseñanza del resto de lenguas a través de programas que garanticen la inmersión en cada cultura idiomática.

El paradigma educativo que defendemos radica en el hecho de concebir la adquisición de una lengua como el acceso a una cultura, como la inmersión en una forma distinta de entender el mundo y las formas de vida, algo que se logra superando el enfoque gramatical propio de las aulas convencionales (QUILES, 2006; FUENTES, 2013; QUILES; CAMPOS, 2015). Es necesario superar la idea tradicional de academia de lenguas o clases de idiomas, para caminar hacia un modelo que garantice la integración plena de los estudiantes en la vida cotidiana del ciudadano nativo, esto es, en la cultura de la lengua meta. Ya en los años ochenta se hablaba de la "inmersión lingüística" como un enfoque novedoso y rompedor que comenzaba en Canadá y que sería adoptado progresivamente por el resto de países:

En las clases de inmersión se aprende el idioma de forma incidental, dentro del proceso de aprender a comunicarse, con un profesor que se centra, sobre todo, en el desarrollo de las nuevas ideas y destrezas que el currículum requiere, aunque lo hace en un idioma que es totalmente extraño para la mayoría de los alumnos de la clase. (GENESEE; LAMBERT; HOLOBOW, 1986, p. 27).

Se trataba de garantizar la transmisión de parte del currículum escolar en la lengua meta; la inmersión, por tanto, hacía referencia al aprendizaje de una lengua extranjera pero fuera de ese país extranjero, esto es, se integraba como materia curricular que iba a traspasar el resto de disciplinas. Así, los centros bilingües se han perfilado desde entonces según este concepto: los escolares - sin salir de su contexto nativo - reciben parte de sus aprendizajes a través del idioma extranjero, tanto por vía oral como escrita. Nos sirve de ejemplo el modelo Aprendizaje Integrado de Contenidos y Lenguas (AICLE) (EURYDICE, 2006; PAVÓN, 2009a, 2009b; PAVÓN; ÁVILA, 2009). No obstante, consideramos que la movilidad de los estudiantes es básica e imprescindible. Defendemos la necesidad de que 
el aprendizaje de esa lengua extranjera (LE), que se ha convertido en un foco de interés prioritario en el sistema educativo actual, pueda entenderse en períodos secuenciados dentro de un entorno homóglota, término introducido desde la sociolingüística por Dabène en 1994, que hace referencia a aquellos contextos en los que la lengua que se aprende se adquiere en el país donde se habla. El concepto alude a la reflexión en torno a lo que tendríamos que ver como una lengua segunda (L2) y lo que sería, en realidad, una lengua extranjera (LE). Así lo han explicado autores que, en fechas más recientes, han vuelto a retomar el término homóglota:

Deducimos, pues, que una L2 es aquella que cumple una función social e institucional en la comunidad lingüística en la que se aprende y que la LE es aquella que se aprende en un contexto en el que carece de función social e institucional. (RÍOS, 2014, p. 604).

Todo lo anterior exige un mayor compromiso de las instituciones a la hora de crear programas en favor de esta idea. De igual forma que existe la Beca Cervantes para la movilidad en España para la E.S.O. y el Bachillerato, debieran aumentarse los proyectos orientados la adquisición de las lenguas extranjeras, especialmente en un momento en el que se ha apostado por una educación pública que camina hacia el plurilingüismo. Tenemos algunos ejemplos en los programas para la cooperación on line entre centros escolares, los llamados eTwinning ${ }^{2}$ que forman parte del Erasmus+ de la Unión Europea, o los planes para los intercambios escolares del Ministerio de Educación, Cultura y Deporte. Sin embargo, se necesita seguir insistiendo en la importancia de la movilidad para garantizar la plena inmersión. Sólo cuando viajamos al país extranjero y convivimos durante un tiempo con los ciudadanos nativos, el desarrollo de la competencia comunicativa se hace realmente efectivo. La educación bilingüe en el contexto de origen de los aprendices encuentra su complemento perfecto en la inmersión plena, secuenciada a lo largo de los cursos académicos, en el país de la lengua meta. La becas del Ministerio centran su interés en los niveles de Secundaria, Bachillerato y Educación Superior, mientras que la Primaria queda relegada a un segundo plano. Y decimos esto porque esta movilidad tan necesaria conlleva un respaldo económico que no todas las familias están en disposición de asumir. Es responsabilidad del estado, por tanto, cubrir esta exigencia en la medida de lo posible.

\footnotetext{
${ }^{2}$ Disponible en: $<$ http://etwinning.es/en/>.
} 
Para todo esto, cuando hablamos de la formación de niños y adolescentes inmersos en el país de la lengua extranjera que están aprendiendo, necesitamos la combinación de tres elementos que van a resultar esenciales. Todos ellos, de forma aislada, realizan su papel positivo, pero la confluencia de los tres nos da el resultado perfecto:

\section{a) Centro educativo (school)}

Nos referimos en este apartado a la presencia de un apoyo docente que refuerce y guíe los aprendizajes que, en sociedad, el estudiante va a ir adquiriendo. Superamos, pues, la idea de clase particular o de academia de idiomas, puesto que la asistencia a la "escuela" no se realiza de manera aislada o descontextualizada en un marco ajeno a la lengua meta, sino todo lo contrario. Si dicha "escuela" está vinculada a los dos elementos siguientes, el proceso será una garantía de éxito porque las destrezas comunicativas serán desarrolladas de una manera mucho más efectiva.

\section{b) Actividades lúdicas y monitores (activity leaders)}

Se realizan actividades diversas, desde deportes hasta excursiones, visitas guiadas o danza. En cada uno de esos momentos los monitores tienen la capacidad de dinamizar procesos favorables al aprendizaje en la propia acción; esto es, el estudiante no está siendo consciente de que está aprendiendo una lengua, sino que a través de su necesidad de uso está adquiriendo competencias que luego procesará como un código lingüístico en las sesiones con el profesorado. Por lo tanto, los monitores constituyen un agente muy importante en el proceso didáctico, puesto que suponen un puente hacia la dimensión sociocultural de la lengua. Todas las actividades que los aprendices van realizando pueden secuenciarse de acuerdo a unas finalidades educativas que, de manera lúdica e inconsciente, se van a ir adquiriendo. Por citar un ejemplo, volvemos la vista a las excursiones propuestas por el centro Country Cousins School ${ }^{3}$, que merece

\footnotetext{
${ }^{3}$ Hemos de decir que el centro Country Cousins School tiene una vinculación activa con la Universidad de Almería que se inició con un contrato de investigación ya concluido, y continuó con su participación en los convenios de intercambio Empresa-Universidad (Fundación Mediterránea). Además, existe un contacto fluido con miembros del Grupo de Investigación CEOM mediante periódicas estancias de investigación. Todo ello es un claro ejemplo de transferencia del conocimiento.
} 
nuestra atención al haber sido galardonado con un Premio ElTons. Nos hemos permitido partir de distintas visitas y rutas que se incluyen en sus programas para edificar la secuencia pedagógica que consideramos se deriva de cada una de ellas. Lo hemos sintetizado de la siguiente forma ${ }^{4}$ :

\begin{tabular}{|c|c|c|c|}
\hline $\begin{array}{l}\text { LUGAR DE } \\
\text { VISITA }\end{array}$ & DESCRIPCIÓN DE LA RUTA & $\begin{array}{l}\text { FINALIDADES EDUCATIVAS / } \\
\text { DESARROLLO DE } \\
\text { COMPETENCIAS }\end{array}$ & $\begin{array}{l}\text { MATERIAL DE } \\
\text { APOYO }\end{array}$ \\
\hline Senderismo & $\begin{array}{l}\text { Los estudiantes hacen } \\
\text { una ruta de senderismo } \\
\text { en el pueblo costero de } \\
\text { Ilfracombe. Desde lo más } \\
\text { alto, pueden tomarse } \\
\text { fotografías del paisaje con } \\
\text { las que, más tarde, partici- } \\
\text { parán en un concurso fo- } \\
\text { tográfico en el que habrán } \\
\text { de describirla y defenderla } \\
\text { ante sus compañeros. }\end{array}$ & $\begin{array}{l}\text { - Garantizar la interacción oral } \\
\text { entre los estudiantes } \\
\text { - Comprender instrucciones } \\
\text { básicas sobre el transcurso } \\
\text { de la actividad } \\
\text { - Ampliar vocabulario relacio- } \\
\text { nado con la flora y la fauna } \\
\text { propia del entorno } \\
\text { - Reforzar destrezas comuni- } \\
\text { cativas para intervenir en } \\
\text { juegos de grupo }\end{array}$ & $\begin{array}{l}\text { Mapa del Sitio } \\
\text { Instrucciones de } \\
\text { ruta } \\
\text { Folleto informativo } \\
\text { (flora y fauna) }\end{array}$ \\
\hline Lee Bay & $\begin{array}{l}\text { Excursión a la playa de Lee } \\
\text { Bay }\end{array}$ & $\begin{array}{l}\text { - Garantizar la interacción oral } \\
\text { entre los estudiantes } \\
\text { - Ampliar vocabulario relacio- } \\
\text { nado con el entorno marino } \\
\text { y la zona costera } \\
\text { - Adquirir habilidades para } \\
\text { describir el paisaje y narrar } \\
\text { la experiencia }\end{array}$ & $\begin{array}{l}\text { Mapa del sitio } \\
\text { Instrucciones de } \\
\text { ruta } \\
\text { Folleto informativo } \\
\text { (historia del lugar, } \\
\text { servicios que ofrece) }\end{array}$ \\
\hline $\begin{array}{l}\text { Visita a } \\
\text { Arlington } \\
\text { Court }\end{array}$ & $\begin{array}{l}\text { Visita a una casa de la épo- } \\
\text { ca victoriana, que aporta } \\
\text { gran conocimiento sobre } \\
\text { la vida y la cultura de las } \\
\text { clases pudientes de esta } \\
\text { época. }\end{array}$ & $\begin{array}{l}\text { - Garantizar la interacción oral } \\
\text { entre los estudiantes y el per- } \\
\text { sonal laboral de la casa museo. } \\
\text { - Comprender textos explicati- } \\
\text { vos propios de la lengua escri- } \\
\text { ta en el contexto del museo } \\
\text { - Entrar en contacto con el } \\
\text { lenguaje propio de la época } \\
\text { victoriana a través de los } \\
\text { nombres de la ropa y los } \\
\text { medios de transporte } \\
\text { - Desarrollar la capacidad para } \\
\text { describir el lugar de la visita }\end{array}$ & $\begin{array}{l}\text { Dosier informativo } \\
\text { Cuestionario con } \\
\text { preguntas sobre ele- } \\
\text { mentos de la casa } \\
\text { Juego de pistas }\end{array}$ \\
\hline
\end{tabular}

\footnotetext{
${ }^{4}$ Modelo secuenciado por nosotros a partir del programa Descovering Devon, ofertado por esta institución educativa, el cual podría trasladarse a otros programas similares de aprendizaje basado en comunidades homóglotas.
} 


\begin{tabular}{|c|c|c|c|}
\hline $\begin{array}{l}\text { LUGAR DE } \\
\text { VISITA }\end{array}$ & DESCRIPCIÓN DE LA RUTA & $\begin{array}{c}\text { FINALIDADES EDUCATIVAS / } \\
\text { DESARROLLO DE } \\
\text { COMPETENCIAS }\end{array}$ & $\begin{array}{l}\text { MATERIAL DE } \\
\text { APOYO }\end{array}$ \\
\hline $\begin{array}{l}\text { Butlins } \\
\text { aquatic park }\end{array}$ & $\begin{array}{l}\text { Día de juegos en el Parque } \\
\text { Acuático Butlins, guiado } \\
\text { por animadores. }\end{array}$ & $\begin{array}{l}\text { - Garantizar la interacción oral } \\
\text { entre los estudiantes } \\
\text { - Desarrollar la competencia } \\
\text { comunicativa a través de los } \\
\text { juegos de agua } \\
\text { - Adquirir nuevo vocabulario y } \\
\text { fórmulas hechas } \\
\text { - Reforzar el uso de la cortesía } \\
\text { verbal y las fórmulas de } \\
\text { tratamiento } \\
\text { - Trabajar la capacidad de narrar } \\
\text { las anécdotas de un día com- } \\
\text { pleto (de forma oral y escrita) }\end{array}$ & $\begin{array}{l}\text { Mapa del sitio } \\
\text { Folleto de apoyo } \\
\text { (Preguntas frecuen- } \\
\text { tes, Normas de } \\
\text { conducta } \\
\text { Lista de vocabulario } \\
\text { y frases hechas }\end{array}$ \\
\hline $\begin{array}{l}\text { Playa de } \\
\text { Wolacombe }\end{array}$ & $\begin{array}{l}\text { Excursión a la playa de } \\
\text { Wolacombe, zona de } \\
\text { referencia para surfistas } \\
\text { en el condado de Devon. } \\
\text { Concurso de castillos de } \\
\text { arena. }\end{array}$ & \begin{tabular}{|l|} 
- Garantizar la interacción oral \\
- entre los estudiantes \\
- Desarrollar la competencia \\
comunicativa a través de los \\
juegos de agua \\
- Adquirir nuevo vocabulario \\
relacionado con la playa y \\
los deportes acuáticos \\
- Entender discursos instructi- \\
vos y expositivos
\end{tabular} & $\begin{array}{l}\text { Texto instructivo. } \\
\text { Normas del con- } \\
\text { curso } \\
\text { Texto informativo } \\
\text { sobre la zona }\end{array}$ \\
\hline $\begin{array}{l}\text { Combe } \\
\text { Martin } \\
\text { wildlife park } \\
\text { Visita al Zoo }\end{array}$ & $\begin{array}{l}\text { Visita al zoo situado en } \\
\text { Combe Martin }\end{array}$ & $\begin{array}{l}\text { - Garantizar la interacción oral } \\
\text { entre los estudiantes } \\
\text { - Ampliar el vocabulario en } \\
\text { torno al mundo animal, sus } \\
\text { hábitos y características } \\
\text { - Comprender textos explicati- } \\
\text { vos propios de la lengua es- } \\
\text { crita en el contexto del zoo: } \\
\text { carteles, folletos explicativos } \\
\text { e información por parte de } \\
\text { los cuidadores de animales }\end{array}$ & $\begin{array}{l}\text { Mapa del sitio } \\
\text { Texto informativo } \\
\text { con puntos de } \\
\text { interés y horarios de } \\
\text { talleres/encuentros } \\
\text { con cuidadores } \\
\text { Lista de vocabu- } \\
\text { lario y preguntas } \\
\text { frecuentes }\end{array}$ \\
\hline $\begin{array}{l}\text { Visita Parque } \\
\text { Nacional de } \\
\text { Exmoore }\end{array}$ & $\begin{array}{l}\text { Ruta de senderismo a } \\
\text { través del paraje natural } \\
\text { del Parque Nacional de } \\
\text { Exmoore }\end{array}$ & $\begin{array}{l}\text { - Ampliar el vocabulario en } \\
\text { torno a los espacios naturales } \\
\text { - Comprender mapas e indi- } \\
\text { caciones }\end{array}$ & $\begin{array}{l}\text { Mapa del sitio } \\
\text { Dossier informativo } \\
\text { sobre la flora y la } \\
\text { fauna del parque }\end{array}$ \\
\hline $\begin{array}{l}\text { Visita a } \\
\text { Lynton \& } \\
\text { Lynmouth }\end{array}$ & $\begin{array}{l}\text { Visita a dos pueblos, unidos } \\
\text { por un teleférico de cremal- } \\
\text { lera movido por agua. }\end{array}$ & \begin{tabular}{|c|} 
- Desarrollar las habilidades \\
para desenvolverse en co- \\
mercios y lugares de ocio
\end{tabular} & $\begin{array}{l}\text { Dossier turístico de } \\
\text { los pueblos visitados }\end{array}$ \\
\hline
\end{tabular}


c) Host families

Ahora bien, quienes tienen un papel fundamental en este último sentido son las familias de acogida. Su función es reforzar la adquisición de la lengua y ser pieza clave en la inmersión cultural. El estudiante puede trasladar todas las habilidades relacionadas con el conocimiento de la lengua-la gramática / el sistema normativo- al uso en contexto, a la interacción cotidiana tanto oral como escrita; pensemos en la comunicación entre los habitantes de la casa, la visualización de la televisión, la escucha de la radio, pero también en la lectura de la prensa, la visita al supermercado, la elección del menú en un restaurante durante el fin de semana, entre otras situaciones donde la forma de vida y el haber cultural de la familia se van a poner de manifiesto constantemente.

\section{EL PROYECTO P.A.L.S: MODELO DE INNOVACIÓN EN UN ENTORNO HOMÓGLOTA, PREMIADO POR EL BRITISH COUNCIL}

El British Council es un organismo de gran reconocimiento en Reino Unido al tratarse de una organización internacional centrada en la educación. Cuenta con sucursales en distintas partes del mundo y acredita a los centros para la enseñanza del inglés ${ }^{5}$. En este contexto, la entidad convoca cada año los prestigiosos premios ElTons a la innovación educativa en la didáctica del inglés. Este galardón es un punto de referencia a nivel internacional, tanto es así que cualquier centro, método o docente que haya sido al menos nominado cuenta con todas las garantías de calidad dentro de este ámbito (O'BRIEN, 2002). Por este motivo hemos visto conveniente detenernos en el proyecto P.A.L.S., diseñado, promovido y coordinado por el centro internacional Country-Cousins School (Reino Unido $^{6}$, un modelo de innovación porque concilia dos elementos de gran actualidad y efectividad en la práctica educativa: la tutoría entre iguales y el llamado aprendizaje servicio (APS).

\footnotetext{
${ }^{5}$ Pueden consultarse las páginas http://www.britishcouncil.org y http://www.britishcouncil.es para buscar más información.

${ }^{6}$ El centro Country-Cousins School está dirigido por J.M. Brinkmann y la coordinadora general del proyecto P.A.L.S. es M. Cabrera Molina, Marketing and Welfare Director. Para más información ver http://www.country-cousins.com.
} 


\subsection{P.A.L.S. como per tutoring y aprendizaje servicio}

Este programa se basa en la inmersión del alumnado extranjero en los centros de secundaria próximos a la zona; esto es, adolescentes de distintas nacionalidades cursan durante un año sus estudios reglados en pleno contexto británico. Es en este marco cuando su equipo docente diseña el proyecto P.A.L.S., que viene a centrarse en la promoción de lo que ellos llaman aceptación de las lenguas entre los estudiantes. Dicho de otra forma, uno de los objetivos del programa era romper los prejuicios que la comunidad de acogida presentaba hacia las lenguas de los jóvenes extranjeros que llegaban para aprender inglés. En un contexto rural en el que los adolescentes de muy diversas procedencias van a convivir durante un tiempo con los estudiantes nativos, ¿Por qué no aprovechar a ese alumnado nativo para hacer de mediadores hacia la adquisición de la lengua? ¿Por qué no buscar una forma de acabar con los estereotipos culturales adquiridos en torno a las lenguas distintas a la nuestra? Se trataba de retomar las conocidas per tutoring (TOPPING, 2015) - tutorías entre iguales - a para ponerlas al servicio de nuestros intereses académicos. Por un lado, el proyecto suponía la creación de una figura de mediador - o tutor - a través del nativo, que sería el guía para el aprendiz extranjero y, al mismo tiempo, se le brindaba una oportunidad a los jóvenes locales de interactuar con niños y niñas procedentes de diferentes países, conocer sus lenguas y sus diversas formas de entender el mundo. En medio de una escuela diversa se nos exige

[...] un cambio de método que debe responder a una metodología combinada y activa con el fin de lograr la participación (se puede trabajar por proyectos - ABP - aplicando el aprendizaje cooperativo - AC -, el aprendizaje entre iguales, las dinámicas de grupo o la enseñanza multinivel - EM - , entre otros. (MARTíNEZ EZQUERRO, 2016, p. 48).

Los entornos plurilingües - o multilingües-, no sólo son contextos donde conviven distintos códigos lingüísticos, sino donde conviven distintas formas de ver el mundo, lo cual propicia situaciones de interculturalidad que, en el ámbito educativo, han de llevar a reconocer la identidad del otro sin anular la propia, al tiempo que manifiesto mi identidad para que el otro la comprenda. Pensemos que las propias convenciones sociales a la hora de saludar, de disculparse, de hacer una petición o de manifestar los afectos, entre otras, son acciones diarias que se 
actualizan en el lenguaje y que vienen determinados culturalmente ${ }^{7}$. Cuando es el adolescente quien entra a formar parte de esta realidad es muy importante su función como mediador en este sentido, para que su identidad lingüística respete la identidad del extranjero y tienda puentes hacia su aprendizaje. Es necesario que los educadores realicen la labor de formar a los estudiantes en valores cívicos que han de comenzar por esa empatía que va a evitar conflictos. Así, podemos decir que la función de estos tutores estaría relacionada con el llamado aprendizaje servicio:

El aprendizaje-servicio (APS) es una metodología orientada a la educación para la ciudadanía, inspirada en las pedagogías activas y compatibles con otras estrategias educativas. El APS es un método para unir éxito escolar y compromiso social: aprender a ser competentes siendo útiles a los demás. (BATLLE, 2011, p. 51).

Lo que en el fondo se está retomando con esta iniciativa es el concepto de comunidades de aprendizaje, vinculando el proceso de adquisición de una lengua por parte de un primer agente - en este caso el estudiante extranjero- con el desarrollo de competencias ciudadanas por parte de un segundo agente - que será el estudiante nativo. Podemos decir que:

El aprendizaje dialógico se produce en interacciones igualitarias donde se reconoce la Inteligencia Cultural de todas las personas, y estas relaciones se orientan hacia la transformación del contexto y de los niveles previos de conocimiento. El aprendizaje dialógico se produce en aquellas interacciones que incrementan la dimensión instrumental del aprendizaje, donde se favorece la creación de sentido, donde se crean relaciones solidarias y finalmente donde igualdad y diferencia se entrelazan. (BOTTON, 2009, p.12).

Sobre los antecedentes en las comunidades de aprendizaje, realizan un estudio muy interesante, Racionero y Serradell (2005), en el que ponen el punto de partida en 1978 a través de la Escuela de Personas Adultas de Verneda (SantMarti, Barcelona), algunos centros del País Vasco y las experiencias de Slavin, Levin i Cormer en Estados Unidos. Es éste un tema abordado también por otros especialistas como Puig (2006) o Rodríguez y Racionero (2015), entre otros, ya que la vinculación del APS con las comunidades de aprendizaje es indudable; sólo podemos partir de un enfoque en el que el servicio a los demás sea el vehículo

\footnotetext{
${ }^{7}$ Recordemos la novela La tesis de Nancy, muy significativa en este sentido.
} 
del aprendizaje si sacamos a la escuela del edificio y la trasladamos al entorno social y hacemos de ella una comunidad en la que eduquemos de manera real y en contexto. Todo esto supone apostar por un cambio, por eso,

[...] tras la decisión de emprender esta transformación, toda la comunidad educativa desarrolla el "sueño», es decir, el ideal de escuela en el que desea transformarse. Tras establecer las prioridades desde esta transformación, se planifica cómo conseguirlas. En todo este proceso toman parte tanto el profesorado y el alumnado como los familiares y vecinos del entorno. (VALLS; GARCÍA, 2015).

El proyecto P.A.L.S., por lo tanto, obedece a un concepto avanzado de lo que implica el acto de educar, porque parte de un enfoque holístico que atiende no sólo a contenidos conceptuales, sino también actitudinales. Educar no es instruir, sino formar ciudadanos que, al tiempo de ser personas, pueden ir acumulando destrezas y conocimientos. Y es que aprender una lengua es también aprender a compartir un comportamiento social; es más, nuestros usos comunicativos nos ubican socialmente y hablan de nuestro acervo cultural, del modo en que se configura nuestro pensamiento. Ya desde la filosofía del lenguaje autores como Wittgenstein nos habían recordado que los límites de nuestro mundo son los límites de nuestra lengua. Si insistimos después de tantos años en que hay que educar para la vida, en que hay que educar no para las aulas sino para la realidad social y la proyección laboral, crear espacios basados en una comunidad de aprendizaje se convierte hoy - sin ser nada nuevo - en una exigencia de vanguardia. De ahí la relevancia de este programa premiado, porque ha sabido trasladar esta filosofía al ámbito de la enseñanza de lenguas, en un entorno homóglota, pero dentro de una comunidad en la que los aprendizajes van a ser diversos y pluridireccionales. La educación en valores, las Ilamadas asignaturas invisibles o la interdisciplinariedad, serán elementos que van a estar presentes en un currículum abierto, que escapa a la concepción de una enseñanza - en este caso de lenguas - tradicional.

\subsection{La organización del programa P.A.L.S.}

El planteamiento general de P.A.L.S. ha sido expuesto en el apartado anterior, de forma que se han puesto de manifiesto las claves que han hecho de él un proyecto innovador. Su organización se articula en torno a tres pilares básicos, que pueden resumirse de la siguiente forma: 


\subsubsection{Selección del grupo P.A.L.S.}

El equipo docente de Country-Cousins establece una selección de estudiantes nativos, matriculados en los institutos de Secundaria y Bachillerato del lugar, para que sean tutores/as de aprendices extranjeros, procedentes del acuerdo de acogida firmado con Country-Cousins. Estos nativos - conocidos como P.A.L.S.- serán los que actúen como mediadores para garantizar la aceptación/ integración de los recién llegados en el entorno sociocultural y académico en el que van a adquirir la lengua meta. Se hace efectiva la tutoría entre iguales - peer tutoring-, de la que ya hemos hablado, así como entra en juego el mencionado aprendizaje servicio. En realidad se está generando una comunidad de aprendizaje articulada a favor de una doble finalidad educativa: la adquisición de una lengua - por una parte- y los valores ciudadanos - por otra.

\subsubsection{Interacción continúa entre el tutor/a P.A.L.S. y el estudiante extranjero}

El tutor P.A.L.S. se convierte desde el comienzo - y siempre desde la concepción del voluntariado- en la persona de referencia para el estudiante de acogida en la comunidad. Lo guía en su integración social y le sirve de mediador en las dificultades, al tiempo que supone un apoyo para su aprendizaje comunicativo. EI P.A.L.S acude a todos los actos organizados por Country-Cousins para sus estudiantes y es partícipe del tiempo de ocio de su tutorizado. Es el enlace entre este y el entorno de acogida. Además, la propia sigla P.A.L.S.- Promoting Aceptans Language Students- coincide con un término coloquial de uso frecuente entre los jóvenes que puede traducirse como colega. El juego de palabras asocia desde el inicio este programa de inmersión lingüística con el enfoque metodológico conferido.

\subsubsection{Colaboración con el Servicio de Juventud Local y la Devon \& Cornwall Policía}

Este aspecto fue uno de los más importantes para la puesta en marcha del programa. La policía local detectaba a menudo conflictos xenófobos entre adolescentes nativos y extranjeros. Muchos de ellos procedían de la ausencia de empatía con la lengua/cultura del otro. La idea de invasión y de ataque a la identidad persistía en muchos de los jóvenes que rechazaban a sus iguales por el 
hecho de hablar otra lengua; además, se detectaba un sentimiento de superioridad lingüística de los unos sobre los otros. La implicación de los P.A.L.S vino a resolver esta situación porque se había puesto en marcha una actuación de aprendizajeservicio vinculada con la enseñanza de lenguas y por iniciativa de un centro educativo. Hablar de multiculturalidad - interculturalidad - que entornos como el nuestro no es ya nada novedoso; sin embargo, en una zona como el norte de Devon y concretamente en un pueblo costero caracterizado por un gran turismo nacional, la situación cambia. Y a la hora de adquirir una lengua - y de pretender enseñarla- ser conscientes de la dimensión cultural y de los prejuicios que todo ello acarrea es un eslabón fundamental sin el cual no vamos a lograr el éxito. En este sentido, P.A.L.S vino a sellar ese eslabón que cerraría la brecha cultural entre las nuevas generaciones.

\section{A MODO DE CONCLUSIÓN}

Cuando hablamos de inmersión lingüística, a menudo pensamos en educación bilingüe, en la elaboración de programas curriculares en una lengua extranjera. Sin embargo, la reflexión que hemos expuesto a lo largo de este trabajo nos pone ante la necesidad de trasladarnos a entornos homóglotas en los que el concepto de inmersión va mucho más allá porque supone adentrarse de forma real y efectiva en la cultura idiomática de la lengua meta. Se ha puesto de relieve la necesidad de implicación institucional para que la movilidad de estudiantes se potencie e incentive en los niveles de secundaria y se extienda a la etapa primaria de forma progresiva. Hemos reformulado, además, el concepto de innovación educativa, concebida desde la visión humanista que no lo vincula necesariamente al uso de las TIC, sino a la implantación de metodologías activas que, desde polos muy diversos, potencien el aprendizaje significativo y pluridireccional.

El siglo XXI pone ante nosotros nuevos desafíos (CONDITO, 2015) para la enseñanza de lenguas. Es el caso del programa educativo P.A.L.S., en el cual confluyen la tutoría entre iguales y el aprendizaje servicio en el marco de las comunidades de aprendizaje. La efectividad del programa se ha hecho patente en unos resultados altamente satisfactorios. Al potenciar la integración de los estudiantes extranjeros desde la implicación de la comunidad nativa, los conflictos se han reducido hasta el punto de no registrarse incidencias graves derivadas del 
choque cultural; es más, lejos de hablar de "choque cultural" se ha pasado a hablar de "confluencia de culturas". Además, el acceso a la lengua extranjera se ha visto doblemente potenciado: de un lado el idioma en su registro formal o académico, propio de las aulas, y por otro en su registro coloquial, propio de las relaciones afectivas en el grupo de compañeros donde el aprendiz se ha visto inmerso. La puesta en marcha de este programa desde 2010 ha supuesto poner en valor la dimensión social del lenguaje más inmediata en contextos multilingües, esto es, el proceso didáctico ha sido realmente un agente de cambio y trasferencia desde las aulas a la propia vida.

\section{REFERÊNCIAS}

BATLLE, R. De qué hablamos cuando hablamos de aprendizaje-servicio? Crítica, v. 61, n. 972, p. 49-54, 2011.

BOTTON, L. de. La sociología dialógica de las comunidades de aprendizaje. RASE, v. 2, n. 3, p. 6-13, 2009.

CONDITO, V. Desafíos para el aula de lengua en el siglo XXI: apuntes para una fundamentación desde una lectura fenomenológica-merlopontiana de la palabra. Álabe, n. 11, 2015. Disponible en <http://revistaalabe.com/index/alabe/article/view/241>.

DABÈNE, L. Repères sociolinguistiques pour l'enseignement des lengues. París: Hachette, 1994.

EURYDICE - La red europea de información sobre educación. Aprendizaje integrado de contenidos y lenguas (AICLE) en el contexto escolar europeo. Madrid: CIDE (Centro de Investigación y Documentación Educativa), 2006.

FUENTES, A. D. Comunicación, lengua e interculturalidad. En: GUERRERO, J. A. (Coord.). Estudios sobre la mediación intercultural. Almería: Servicio de Publicaciones de la Universidad de Almería, 2006, p. 107-34.

GENESEE, F.; LAMBERT, W. E.; HOLLOBOW, N. E. La adquisición de una segunda lengua mediante inmersión: el enfoque canadiense. Infancia y Aprendizaje, n. 33, p. 27-36, 1986.

MARTÍNEZ EZQUERRO, A. Educación lingüística y literaria en el siglo XXI: legislación educativa y atención a la diversidad. En: MARTÍNEZ EZQUERRO, A.; FERNÁNDEZ-FÍGARES, M. C. (Coord.). Cultura en la diversidad: educación lingüística y literaria en las aulas del siglo XXI. Barcelona: Octaedro, 2016. p. 33-66.

O'BRIEN, T. The British Council: promouvir l'apprentissage de l'anglais en Europe. Cahiers de l'Institud de Linguistique de Louvain, v. 28, n. 1-2, p. 207-15, 2002. 
PAVÓN, V. Cambios en la realidad educativa a través de la enseñanza integrada de lengua y contenidos. En: Las lenguas extranjeras como vehículo de comunicación intercultural. Madrid: Ministerio de Educación, 2009a. p. 65-83.

. La introducción de un programa de enseñanza integrada de lengua y contenidos en la educación superior: claves para impartir asignaturas a través de una segunda lengua. En: PAVÓN, V.; ÁVILA, J. (Ed.). Aplicaciones didácticas para la enseñanza integrada de lengua y contenidos (AICLE/CLIL/ÉMILE). Sevilla: Consejería de Educación de la Junta de Andalucía-Universidad de Córdoba, 2009b. p. 29-53.

PAVÓN, V.; ÁVILA, J. (Ed.). Aplicaciones didácticas para la enseñanza integrada de lengua y contenidos (AICLE/CLICL/ÉMILE). Sevilla: Consejería de Educación de la Junta de AndalucíaUniversidad de Córdoba, 2009.

PUIG, P. Los centros de formación por alternancia: desarrollo de las personas y de su medio. 2006. Tesis (Doctorado en Educación) - Universitat Internacional de Catalunya, Barcelona, Espanha, 2006.

QUILES, M. C. El español como lengua extranjera: pautas de actuación docente en una escuela plural. RedELE - Revista Electrónica de Didáctica ELE, v. 3, n. 6, 2006.

QUILES, M. C.; CAMPOS, M. Una aproximación empírica al español escrito por escolares inmigrados. RIEM: Revista Internacional de Estudios Migratorios, v. 5, n. 2, p. 172-200, 2015.

RACIONERO, S.; SERRADELL, O. Antecedentes de las comunidades de aprendizaje. Educar, n. 35, p. 29-39, 2005.

RÍOS, A. El proceso de inmersión lingüística y cultural: un nuevo enfoque orientado a la acción a través de la experiencia. En: CONTRERAS, N. M. (Ed.). La enseñanza del español como LE/L2 en el siglo XXI, ASELE. Alicante : Biblioteca Virtual Miguel de Cervantes, 2014. p. 603-16.

RODRÍGUEZ, M. R.; RACIONERO, S. Una experiencia de aprendizaje-servicio en comunidades de aprendizaje. Profesorado: Revista de Currículum y Formación del Profesorado, v. 19, n. 1, p. 314-33, 2015.

TOPPING, K. Peer tutoring old method, new developments. Infancia y Aprendizaje, n. 38, 2015. Disponible en: <http://www.tandfonline.com/doi/full/10.1080/02103702.20 14.996407>.

VALLS, R.; GARCÍA, C. Comunidades de aprendizaje. DINLE. Diccionario Digital de Nuevas Formas de Lectura y Escritura. 2015. Disponible em: <http://dinle.usal.es/searchword. php?valor=comunidades+de+aprendizaje>. 


\section{Sobre la autora:}

María del Carmen Quiles Cabrera: Es doctora en Filología Hispánica por la Universidad de Almería, Máster en Promoción de la Lectura y la Literatura Infantil (Universidad de Catilla-La Mancha) y tiene el Título de Experto en Español Lengua Extranjera por la Universidad de Murcia. E-mail: qcabrera@ual.es

Recebido em fevereiro de 2018

Aprovado em maio de 2018 